\title{
Guidance and Control of Position and Attitude for Rendezvous and Dock/Berthing with a Noncooperative/Target Spacecraft
}

\author{
Gilberto Arantes Jr. and Luiz S. Martins-Filho \\ Center of Engineering, Modeling and Applied Social Sciences, Federal University of ABC, \\ Avenida dos Estados 5001, 09210-971 Santo André, SP, Brazil \\ Correspondence should be addressed to Luiz S. Martins-Filho; luiz.martins@ufabc.edu.br
}

Received 22 January 2014; Accepted 16 May 2014; Published 6 July 2014

Academic Editor: Vivian Martins Gomes

Copyright (C) 2014 G. Arantes Jr. and L. S. Martins-Filho. This is an open access article distributed under the Creative Commons Attribution License, which permits unrestricted use, distribution, and reproduction in any medium, provided the original work is properly cited.

\begin{abstract}
Noncooperative target spacecrafts are those assets in orbit that cannot convey any information about their states (position, attitude, and velocities) or facilitate rendezvous and docking/berthing (RVD/B) process. Designing a guidance, navigation, and control (GNC) module for the chaser in a RVD/B mission with noncooperative target should be inevitably solved for on-orbit servicing technologies. The proximity operations and the guidance for achieving rendezvous problems are addressed in this paper. The outof-plane maneuvers of proximity operations are explored with distinct subphases, including a chaser far approach in the target's orbit to the first hold point and a closer approach to the final berthing location. Accordingly, guidance solutions are chosen for each subphase from the standard Hill based Closhessy-Willtshire (CW) solution, elliptical fly-around, and Glideslope algorithms. The control is based on a linear quadratic regulator approach (LQR). At the final berthing location, attitude tracker based on a proportional derivative (PD) form is tested to synchronize the chaser and target attitudes. The paper analyzes the performance of both controllers in terms of the tracking ability and the robustness. Finally, it prescribes any restrictions that may be imposed on the guidance during any subphase which can help to improve the controllers tracking ability.
\end{abstract}

\section{Introduction}

In order to perform rendezvous with a spacecraft, key technologies for rendezvous and docking/berthing (RVD) are required, for example, integrated system of robotics, autonomous guidance, navigation and control (GNC) systems, and sophisticated hardware/software capabilities. On-orbit servicing (OOS) is broadly defined as the execution of assembly, repair, upgrade, refuel, rescue, retrieval, and maintenance of a spacecraft in orbit [1]. Table 1 shows possible OOS scenarios based on the status of the satellite $[2,3]$. A compulsory requirement for the service satellite is the ability to deal with a noncooperating client or target satellite. A noncooperating spacecraft is not able to provide information on its own about its position and attitude; moreover, it cannot maneuver to facilitate the RVD process. The problem of RVD with a noncooperative target can be seen as an extension of the problem concerning a cooperative target. However, the focus here is laid upon dealing with noncooperative targets because this problem is quite new and needs to be explored further. In a crude sense, rendezvous with a noncooperating target involves critical tasks such as visual servoing system and grapple task $[4,5]$. An overview of GNC architecture for autonomous rendezvous and docking/berthing is shown in Figure 1. Due to the noncooperative nature of the target, intensive supervision, inspection, and preparation modes for the service spacecraft are required $[6,7]$.

RVD problem with a noncooperating target can be broadly divided into three stages [8]: long-range or phasing, proximity operations, and terminal phase. Each phase has its own mission specific subphases with special initial and terminal conditions to be met $[3,8,9]$. In light of OOS, this work presents suitable control strategies for proximity phase operations in autonomous $\mathrm{RVD} / \mathrm{B}$, without venturing into phasing or final stage docking operations. Realistic subphases for proximity phase operations are proposed and analyzed starting from far range, fly-around inspections, and then a closer approach. 


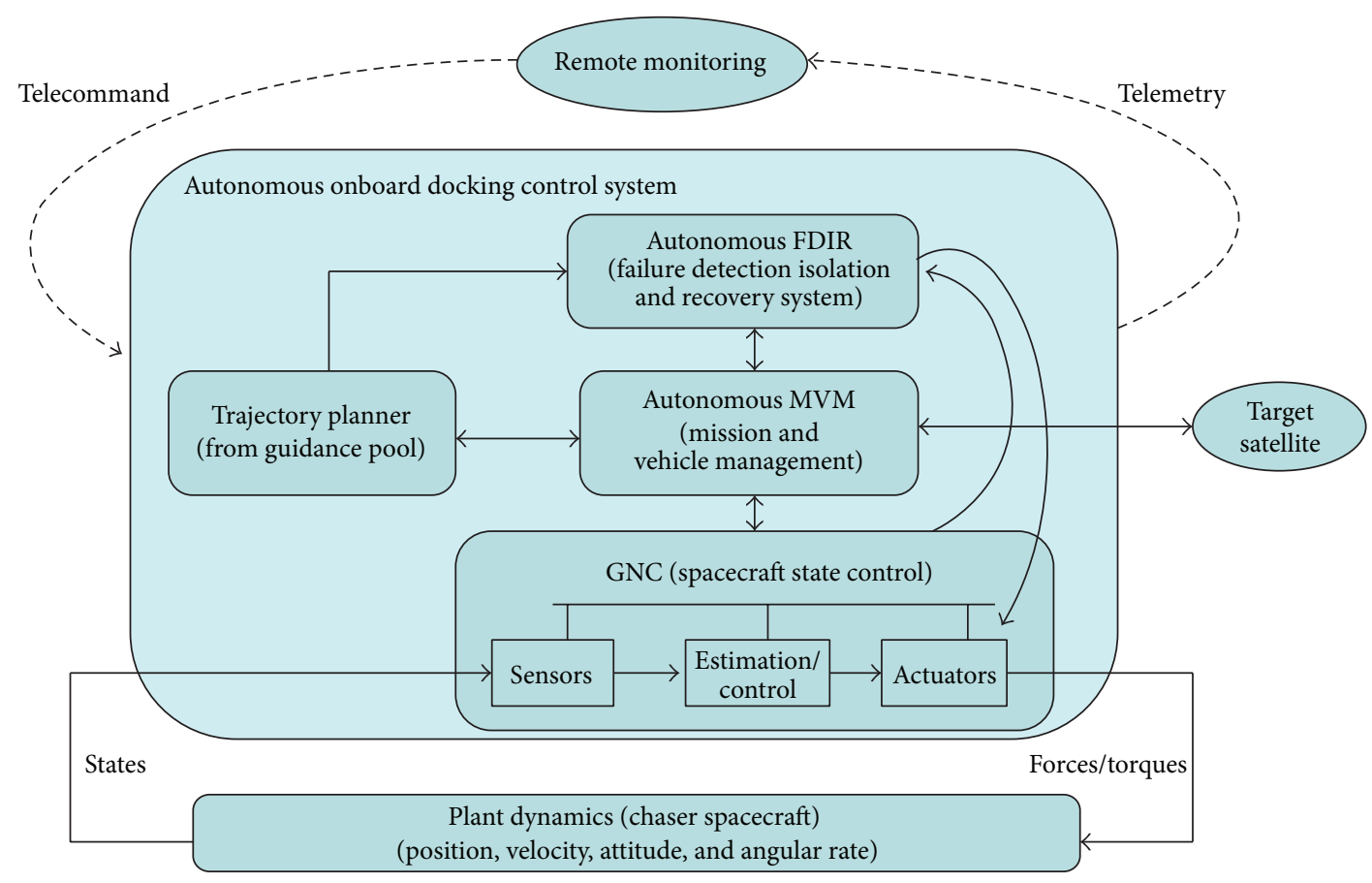

FIGURE 1: GNC architecture for autonomous docking/berthing.

TABLE 1: On-orbit servicing scenarios.

\begin{tabular}{ll}
\hline Status of spacecraft & OOS provided \\
\hline $\begin{array}{l}\text { End of lifetime due to } \\
\text { propellant, all systems } \\
\text { operational }\end{array}$ & $\begin{array}{l}\text { Refuelling in situ to extend } \\
\text { satellite mission lifetime }\end{array}$ \\
Partial failure of satellite & $\begin{array}{l}\text { Replacement/repair of } \\
\text { failed unit }\end{array}$ \\
$\begin{array}{l}\text { Erroneous injection of } \\
\text { satellite }\end{array}$ & $\begin{array}{l}\text { Rescue and reboost to } \\
\text { operational orbit }\end{array}$ \\
$\begin{array}{l}\text { Threat to other space assets } \\
\text { and/or Earth }\end{array}$ & Retrieval and deorbit \\
\hline
\end{tabular}

During proximity operations, the chaser is guided along carefully chosen guidance trajectories towards the target from the available pool of algorithms. A single maneuver to bring the chaser from the entry gate to the final point of capture is rather impossible for many reasons such as chaser's safety, target detection, relative navigation, fuel consumption, and disturbance effects. Furthermore, during these operations, unlike with cooperative targets, the chaser cannot confine its motion to the orbital plane of the target and it should be capable of executing maneuvers out of this plane. This need may arise during target inspections, or for docking/berthing in the final phases, and therefore the chaser should be able to reach any location around the target. Consequently, a disintegrated proximity operational phase is the most favorable option, and the definition of intermediate hold points becomes necessary. This strategy is adopted in this work. From the entry gate and along the path of the chaser towards the target, two hold points $\mathrm{H} 1$ and $\mathrm{H} 2$ (see Figure 2) are defined. And two subphases connecting these different guidance schemes and tracking controller are needed.

This work is organized as follows. Section 2 presents the problem formulation within relative RVD/B dynamics and the tracking controllers for attitude and position. Section 3 presents the study case scenarios for RVD with a noncooperative target, and the performance of the tracking controllers for the proposed RVD scenario. Finally the conclusion and future recommendations are outlined in Section 4.

\section{Problem Formulation}

The problem formulation can be divided into the modeling of $\mathrm{RVD} / \mathrm{B}$ relative dynamics, the position controller design, and the attitude controller design.

2.1. $R V D / B$ Relative Dynamics. In order to develop the equations of relative motion, let $(X, Y, Z)$ represent the inertial frame centered in Earth and let $\mathbf{r}_{c}$ and $\mathbf{r}_{t}$ be the chaser and target positions in inertial frame. Their relative distance denoted by $\mathbf{s}$ is given by

$$
\mathbf{s}=\mathbf{r}_{c}-\mathbf{r}_{t},
$$

and therefore the relative acceleration is

$$
\ddot{\mathbf{s}}=\ddot{\mathbf{r}_{c}}-\ddot{\mathbf{r}}_{t} \text {. }
$$

As most of potential target satellites are in a near circular orbit, we suppose that the angular motion is constant. The general equation of motion under the influence of a central force is given by Newton's law of gravitation:

$$
F_{g}(\mathbf{r})=-G \frac{M m}{r^{2}} \frac{\mathbf{r}}{r}=-\mu \frac{m}{r^{3}} \mathbf{r}
$$




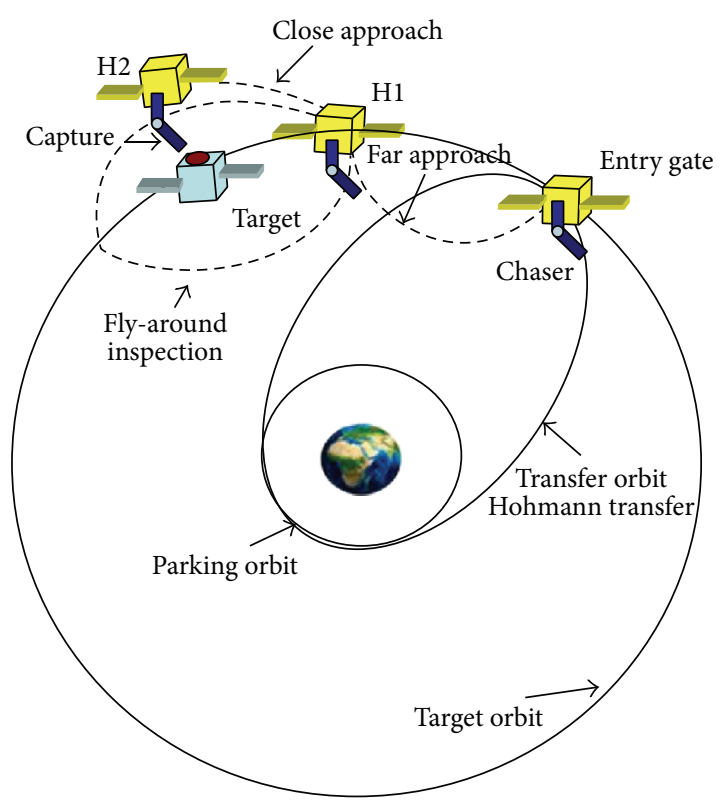

Figure 2: RVD mission scenario showing all three subphases and the hold points during proximity operations. Phases are separated as far range, inspection, and close approach between the entry gate and the hold points $\mathrm{H} 1$ and $\mathrm{H} 2$.

where $F_{g}$ is the gravitational force, $G$ is the universal gravitation constant, $M$ is the mass of the central body (Earth), $m$ is the mass of the spacecraft, $\mathbf{r}$ is the radius vector, and $\mu=$ $G M$. By normalizing the gravitation force, that is, dividing by the mass of the spacecraft, we write the chaser and target equations for the absolute motion in the inertial frame as follows:

$$
\begin{aligned}
& \text { Target: } f_{g}\left(\mathbf{r}_{t}\right)=\ddot{\mathbf{r}_{t}}=\frac{\mathbf{r}_{t}}{r^{3}}, \\
& \text { Chaser: } f_{g}\left(\mathbf{r}_{c}\right)=\ddot{\mathbf{r}_{c}}=\frac{\mathbf{r}_{c}}{r^{3}} .
\end{aligned}
$$

It is convenient to describe the motion with respect to the target frame which is a noninertial frame and therefore we write

$$
\frac{d^{* 2} \mathbf{s}^{*}}{d t^{2}}+\omega \times\left(\omega \times \mathbf{s}^{*}\right)+2 \omega \times \frac{d^{*} \mathbf{s}^{*}}{d t}+\frac{d \omega}{d t} \times \mathbf{s}^{*}+\frac{\mu}{r^{3}} \mathbf{M} \mathbf{s}^{*}=\mathbf{f},
$$

where $\mathbf{s}$ is given in (1) and $*$ denotes that it is described in the target frame, $\mathbf{f}$ is the applied force, and $\omega$ is the orbital motion of the target; that is, the target frame named Hill frame [8] is rotating with angular velocity $\omega$. Linearizing the gravitation force model of chaser spacecraft around the target spacecraft and computing the terms on the left side of (5), we obtain

$$
\begin{gathered}
\ddot{x}-2 n \dot{z}=f_{x}, \\
\ddot{y}+n^{2} \dot{y}=f_{y}, \\
\ddot{z}+2 n \dot{x}-2 n^{2} \dot{z}=f_{z} .
\end{gathered}
$$

These equations are known as Hill equations and they describe the motion of the chaser with respect to the target spacecraft.

For the attitude model it is reasonable to assume that the angular velocities are constant for the target and chaser spacecraft. In this case the relative attitude model is given by [8]

$$
\dot{\mathbf{q}}_{r}=\frac{1}{2} \Gamma\left(\mathbf{q}_{r}\right) \omega_{r}=\frac{1}{2} \Omega\left(\omega_{r}\right) \mathbf{q}_{r},
$$

where

$$
\Omega(\omega)=\left[\begin{array}{cc}
0 & -\omega^{T} \\
\omega & \mathbf{S}(\omega)
\end{array}\right]
$$

the quaternion $\mathbf{q}_{r}$ represents the relative attitude, and $\omega_{r}$ is the relative angular velocity. The quaternion $\mathbf{q}_{r}$ is obtained as [8]

$$
\mathbf{q}_{r}=\mathbf{q}_{c} \otimes \mathbf{q}_{t}^{-1} .
$$

Equations (5) and (7) give the relative model for position and attitude. They are the models used for the attitude and position control design.

2.2. RVD/B Control Design: Position. Optimal controllers constitute a category of controllers designed based on a cost function, a function formulated on the requirements on state variables, control inputs, terminal conditions, time constraints, and so forth. The linear quadratic regulator (LQR) is a quite well-liked approach of optimal controllers with features of fuel efficiency and robustness. For the position control system, the LQR controller is adopted in this work. The dynamic system, that is, the Hill equations, must be given in the state space form:

$$
\dot{\mathbf{x}}=\mathbf{A x}+\mathbf{B u}
$$

Note that the out-of-plane motion $(y)$ is decoupled from the in-plane motion $(x z)$. Therefore the control for $H$-bar $(y)$ consists of a simple SISO system. The problem is to find the control gain $\mathbf{K}$ for the linear state feedback control law $\mathbf{u}=$ $-\mathbf{K x}$ that minimizes the quadratic cost function

$$
J=\int_{0}^{\infty}\left(x^{T} Q x+u^{T} R u\right) d t
$$

with $R=R^{T} \geq 0$ and $Q=Q^{T} \geq 0$. The optimal gain $\mathbf{K}$ which solves the LQR problem is given by

$$
\mathbf{K}=R^{-1} B^{T} S,
$$

where $S$ is unique, positive semidefinite solution to the algebraic Riccati equation

$$
A^{T} S+S A-S B R^{-1} B^{T} S+Q=0 .
$$

The $\mathbf{K}$ matrix can be computed offline. The state weighting matrix $Q$ is chosen to be diagonal with all its entries $10^{-1}$ and the control weighting matrix $R$ is taken to be an identity matrix (or a scalar, for the out-of-plane problem). 


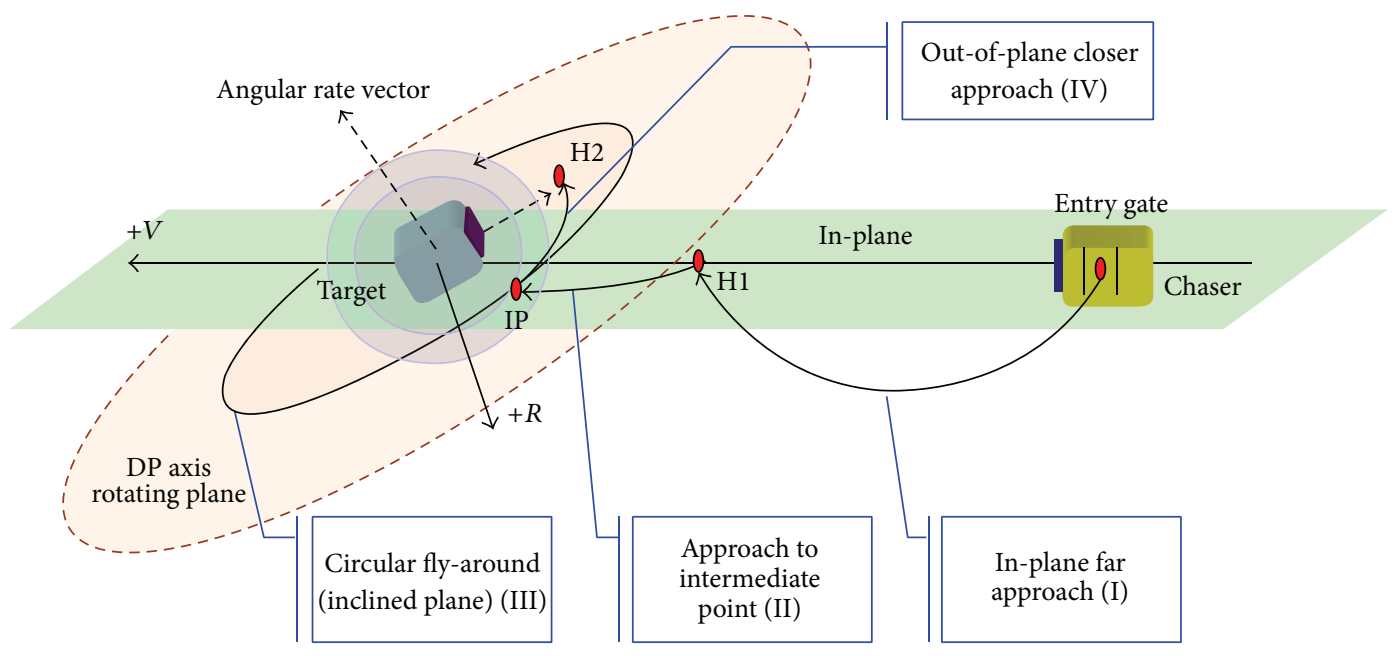

FIGURE 3: RVD scenario with noncooperative target.

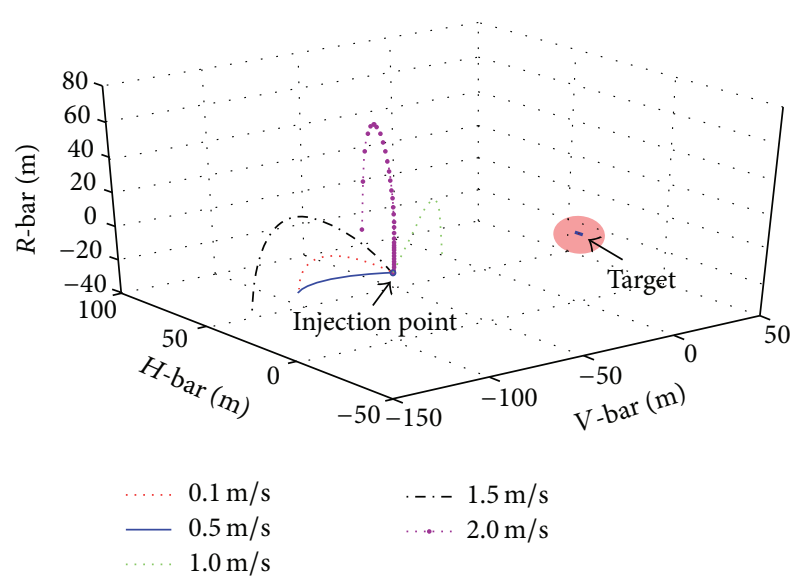

FIGURE 4: Injection errors in position and residual velocities compensation.

2.3. RVD/B Control Design: Attitude. Relative attitude controller is a fundamental component of the GNC subsystem of the chaser dealing with either cooperative or noncooperative targets. Implementation is much simpler when intensive control efforts are not required, such as the case of interaction with cooperative targets due to the absence of navigation issues and time delays in state estimation. With noncooperative targets, the motion of target must be estimated continuously using a navigation filter. Attitude planning for chaser during proximity operations should be to regulate the attitude when target is within LOS of camera and point to target otherwise. Additionally, synchronization, that is, regulating relative attitude and rates near zero, should be done during berthing or prior to an in-line approach for docking. Two different controllers are proposed for the chaser attitude: one for keeping its absolute attitude and another for controlling its relative attitude considering the target. The absolute attitude controller takes into account exclusively the chaser dynamics. This control tool can be used to reorient the chaser any time from its current orientation to a new attitude reference. The attitude tracker can be used for pointing to

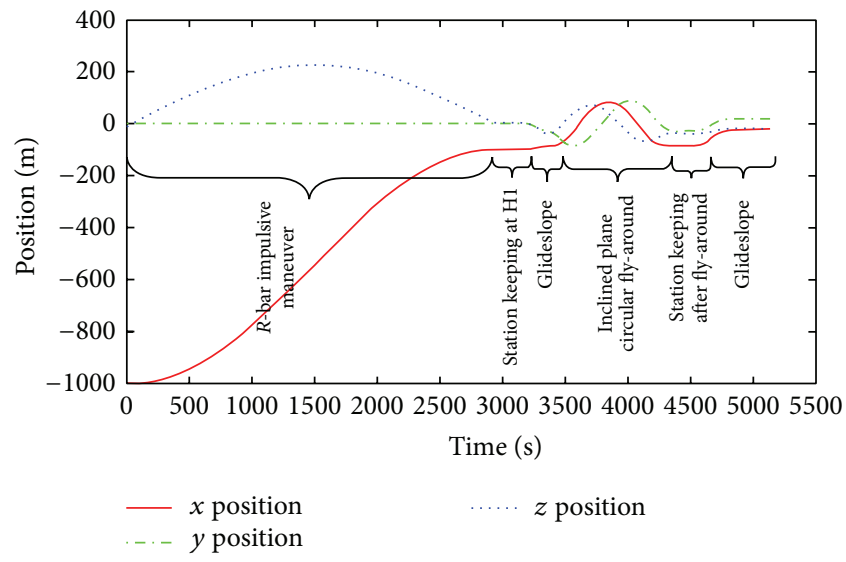

Figure 5: Position profile for different manoeuvres during the target approach.

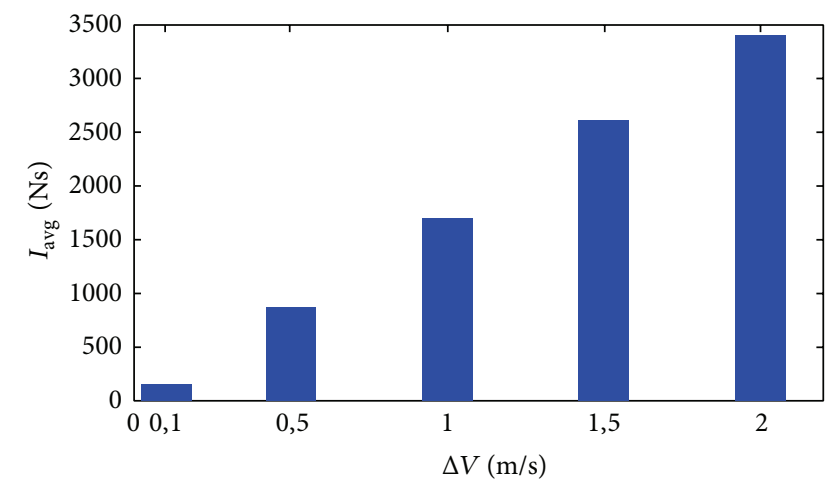

FIgURE 6: Average impulse needed to correct the injection errors.

target from farther distances or during fly-around inspection or even during close approach. It aims to control the line of sight (LOS) of the chaser's camera and to maintain the target in the focus all the time. By enabling chaser target attitude synchronization, the tracking controller is very helpful in understanding the behavior and motion of the target. A linear 

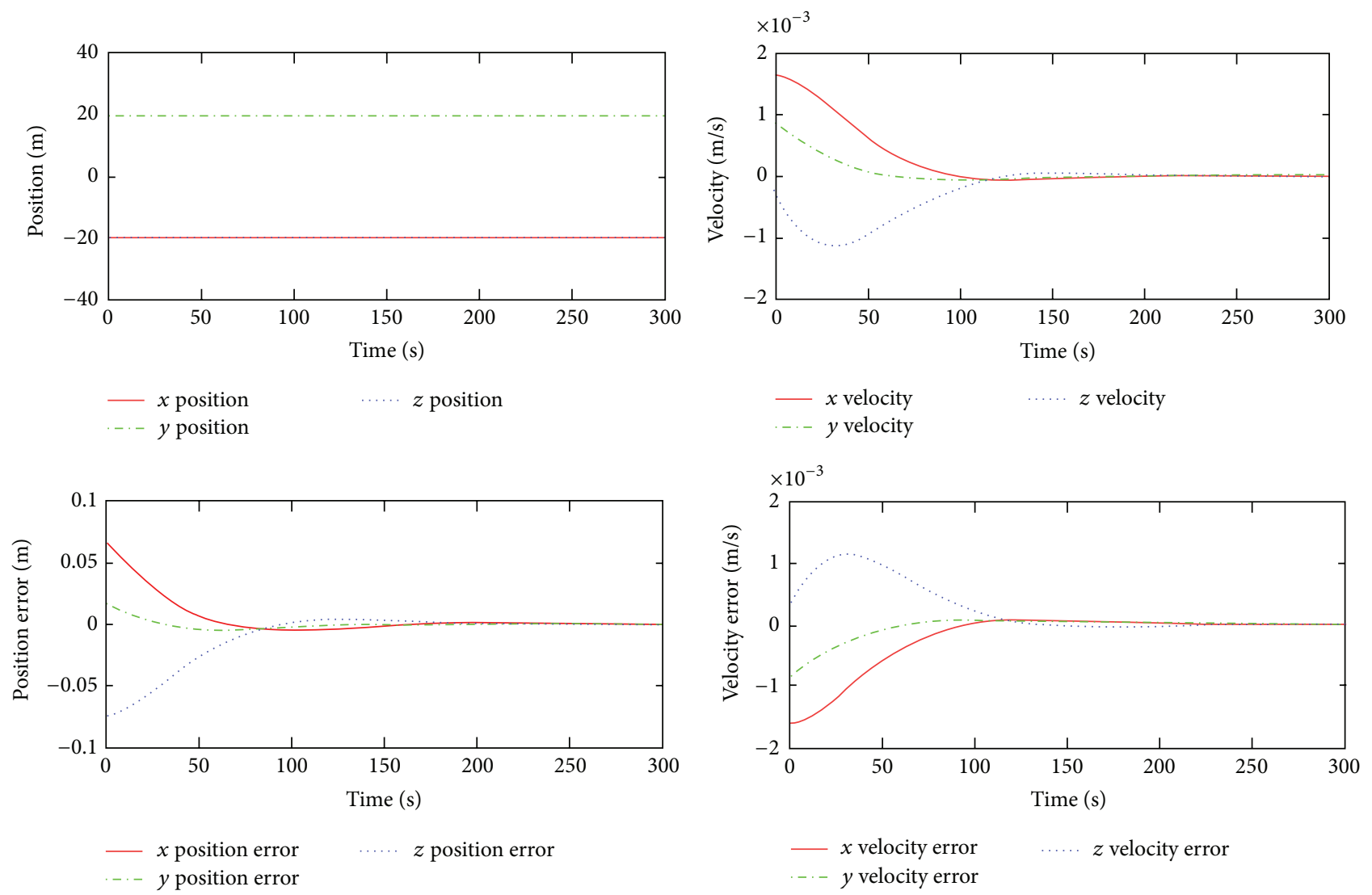

FIGURE 7: Chaser's position profile using LQR controller.

state feedback control law is adopted in the chaser attitude controller. It is given by [8]

$$
\mathbf{u}=K \mathbf{q}_{e}-C \omega_{c}
$$

where $\mathbf{q}_{e}$ is the attitude error quaternion and $\omega_{c}$ is the body angular rate of the chaser. For positive scalar constants from $k_{i}$ and $c_{i}$ the control law is globally asymptotically stable. This control law is suitable for real time implementation as demonstrated by [8].

\section{Simulations and Results}

In this section we present the performance of the position and the attitude controllers. The numerical simulations of the adopted models are also described and discussed here.

3.1. Case Studies. Extensive simulation studies with appropriate selection on maneuvers were conducted for chaser in diverse orbits and mass properties. We will assume that the chaser is equipped with a multiple imaging system with at least two cameras: one for the vision based navigation system, in order to provide pose of the target, and a second one dedicated entirely to assist the final phase manipulator operations. In general terms, it is assumed that the completion of mission is not time-constrained, though these time restrictions may be imposed for some maneuvers during any subphase. However the fuel constraints have priority over the execution time in selecting a particular maneuver. Attitude control is driven and demonstrated by the strategy of target pointing using the navigation camera. All the set of maneuvers during proximity operation is illustrated in Figure 3.

The first hold point $\mathrm{H} 1$ is chosen to be in the target's orbit at $-100 \mathrm{~m}$ along $-V$ bar. Had this point been at a different altitude in plane other than the target's orbit, the chaser would also be subject to a drift because of orbital forces and this drift is even furthered in the presence of any injection errors and residual velocity components. Since out-of-plane velocity component is absent during in-plane motion, if $\mathrm{Hl}$ is chosen to be along target's orbit, low residual in-plane velocity components do not prove very risky. The berthing operation is the more flexible and the preferred option for the GNC system of the chaser in a mission with noncooperative targets. It relaxes the terminal constraints on position, attitude, and their rates to be acquired by the chaser at $\mathrm{H} 2$ and thereby lessens its control efforts. Hence, berthing parameters are chosen to evaluate the performance of the controllers at $\mathrm{H} 2$.

3.1.1. Injection Maneuver: Entry Gate. Position injection error compensation is analyzed during the injection in the entry gate. The result is shown in Figure 4. The LQR controller stabilizes for any residual velocity less than $2 \mathrm{~m} / \mathrm{s}$ (the worst case). 

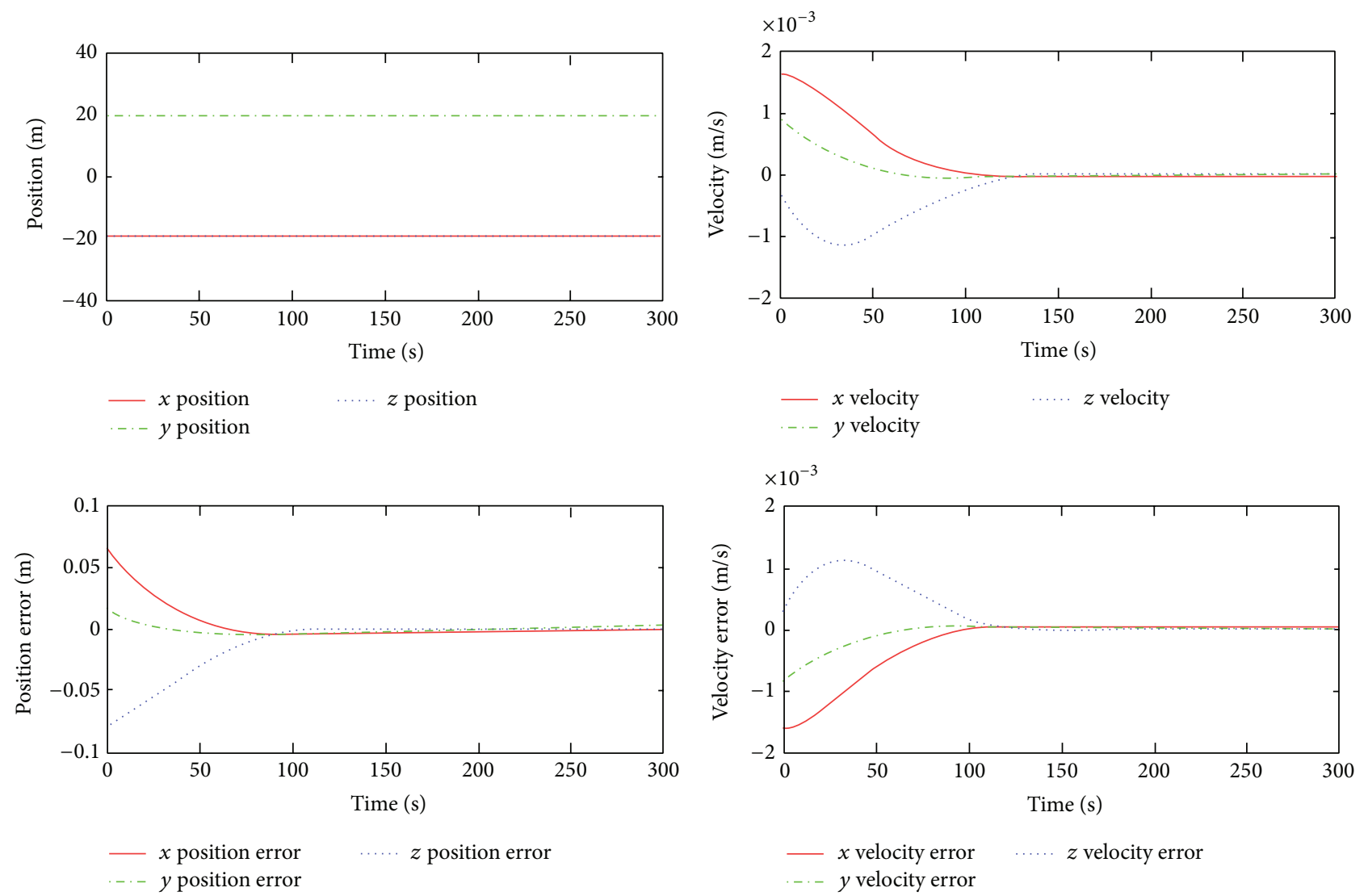

FIGURE 8: Berthing H2 location performance for position and velocity.

The position profile for the different maneuvers performed during the procedure of target approaching can be seen in Figure 5.

The average impulse to bring the chaser back to the nominal $\mathrm{H} 2$ position as a function of the velocity injection errors is shown in Figure 6. The results clearly show that the control efforts are proportional to the injection velocity errors which are assumed to be caused mostly by thrust errors during the transfer.

3.1.2. Transition from Entry Gate to $H 1$ and Inspection Phase. The maneuver from the entry gate to $\mathrm{H} 1$ which is followed by inspection is compulsory for a noncooperating target. The LQR controller performance is shown in Figure 7. The guidance schemes are important either to save propellant, for example, $R$-bar impulsive maneuver, or to bound the flight time.

3.1.3. Final Approach: Berthing. Candidate maneuvers to bring the chaser to $\mathrm{H} 2$ position can be a glideslope or an out-of-plane angular fly-around. Closed loop controlled glideslope maneuver proves to be a better and a standard option because it allows planning on the transfer impulses as well as on the final velocities reachable at H2. A gradual decelerating approach with very low residual velocities makes this approach very safe. On the other hand, angular flyaround needs continuous actuation and thus proves very costly compared to glideslope. After attitude synchronization at $\mathrm{H} 2$, simultaneous $6 \mathrm{DOF}$ control for relative position and attitude should be done if a docking is to be performed via an in-line approach. The controller performance for the final approach is shown in Figure 8. The berthing maneuver is executed successfully. The attitude controller is shown in Figure 9.

The angular velocities results are shown in Figure 10. Note that the chaser is able to rotate at an angular rate of $20 \mathrm{deg} / \mathrm{s}$. This is considered the worst case scenario for a noncooperative target.

\section{Conclusion}

The study on the development of OOS technology is becoming more demanding in light of increasing necessity in satellite servicing or removal of space debris. This kind of missions has to deal with either cooperatively designed future assets or current assets which are extremely noncooperative. Intended to give a realistic approach and address the existing situation in space, this works aims to look into proposing the guidance and control schemes to perform RVD with noncooperative targets. Since the scenarios which encapsulate a noncooperative target are much more complicated than the ones with a cooperative target, the methodology presented in this work can also be suitably molded and adopted for future day friendly designed cooperative targets. Proximity operations 

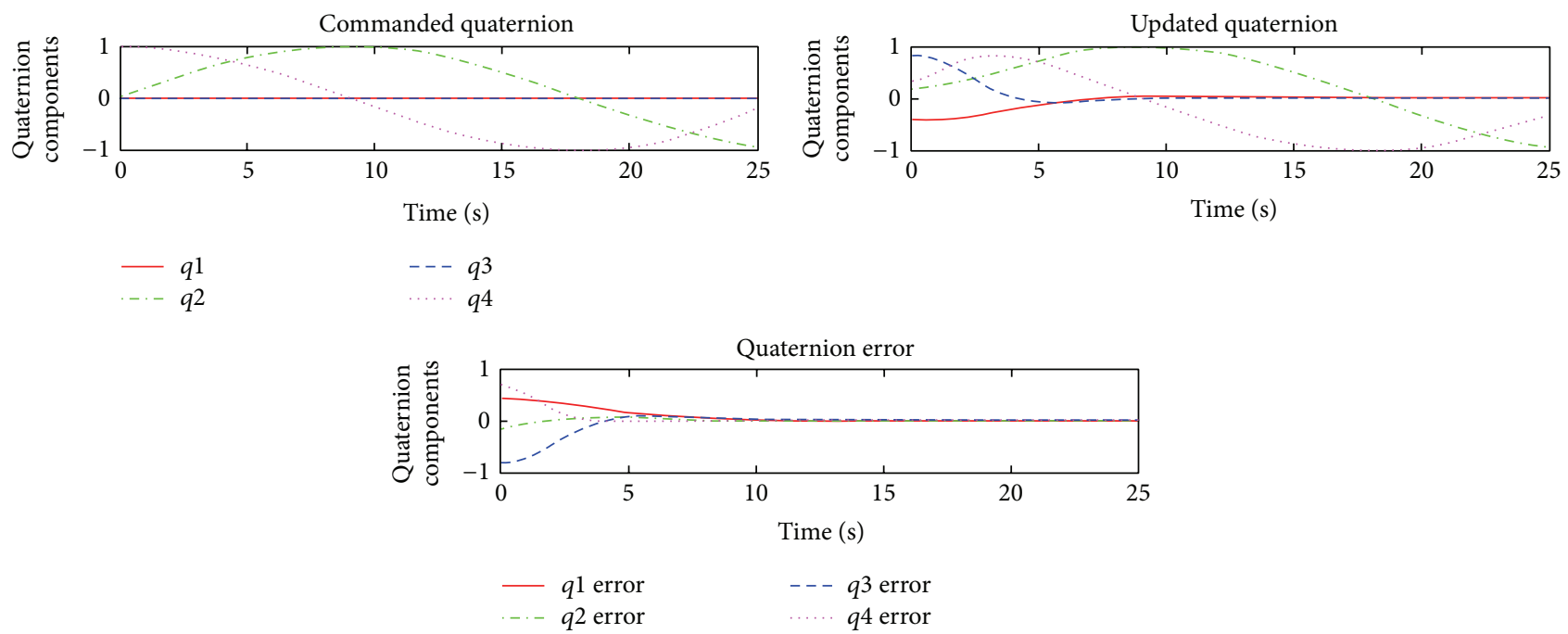

Figure 9: Attitude results and quaternion error at $\mathrm{H} 2$.
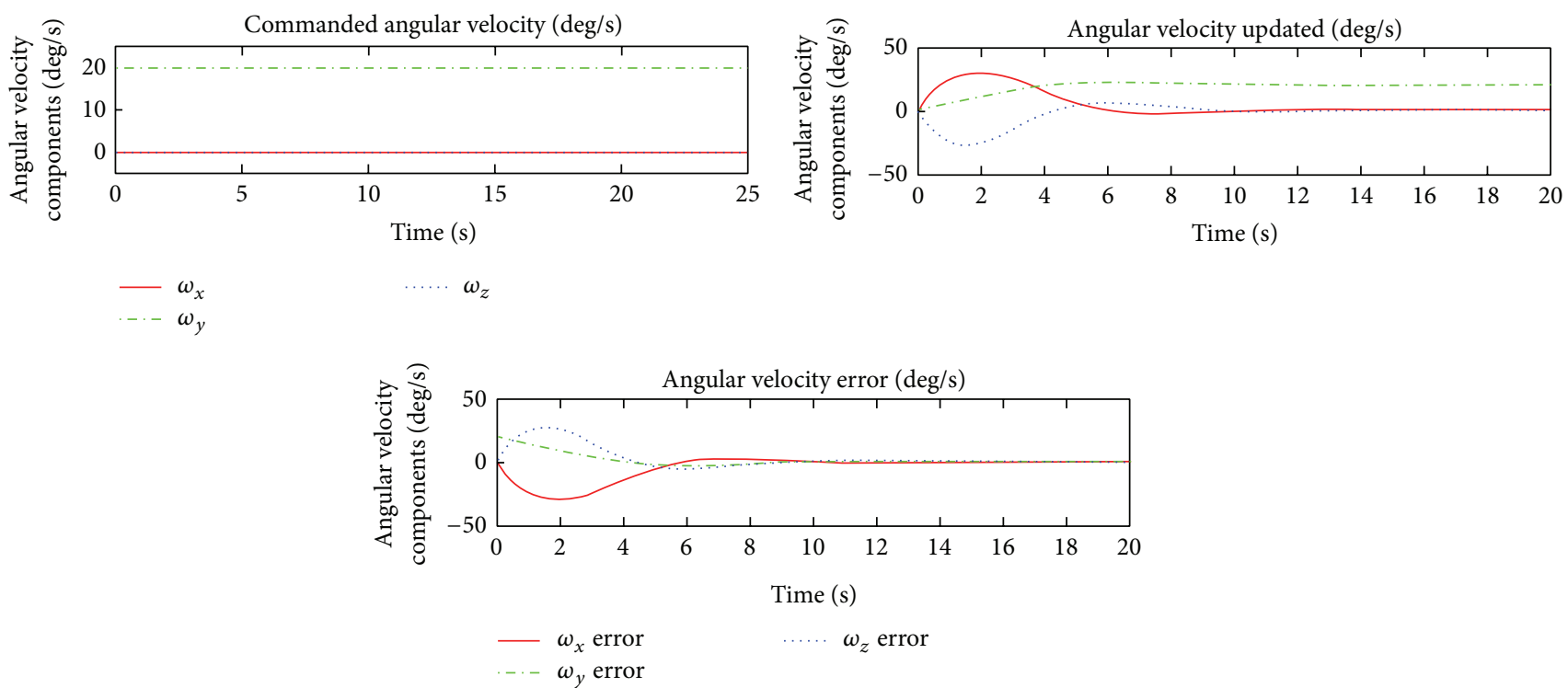

FIgURE 10: Angular rate error at $\mathrm{H} 2$.

play a key role and a careful investigation of this phase with suitable GNC schemes should be done prior to attempting any mission with these targets. These operations are rather more general and common to all RVD missions differing only in the level of complexity they impose depending upon the nature and behavior of the targets. They are therefore much more complicated in missions with noncooperative targets. One advantage is that these operations can be seen independently of phasing maneuvers. A methodology for performing far and close range operations elaborating dynamics and GNC schemes with a generic target is presented in this work. Both set point and tracking controllers based on LQR for position and PD with angular velocity constraints for attitude are successfully tested for the proposed guidance. The attitude controller performance is also verified for high angular rate of the target. Future recommendations are proposed as follows. (i) The safety of the approaches should be further investigated especially for the closer approach.

(ii) Analysis should be extended to include elliptical orbits.

(iii) Control parameters should be retuned for different phases for efficient trajectory tracking with clear geometric mission constraints.

(iv) Elaborate the model to include uncertainties from the navigation solution, external disturbances, and so forth.

(v) Further the study to include grasping from berthing location using capture dynamics subject to additional constraints like fuel, plume impingement, relative position, and velocity constraints. 
(vi) Extend the attitude model to include other actuators that can provide for continuous actuation.

\section{Disclosure}

The authors are the only ones responsible for the printed material included in this paper.

\section{Conflict of Interests}

The authors declare that there is no conflict of interests regarding the publication of this paper.

\section{Acknowledgments}

The authors gratefully acknowledge the financial support from of the Science without Borders Program (CAPES, Brazil) and the University of ABC (São Bernardo do Campo, Brazil).

\section{References}

[1] A. Tatsch, N. Fitz-Coy, and S. Gladun, "On-orbit servicing: a brief survey," in Proceedings of the IEEE International Workshop on Safety, Security, and Rescue Robotics (SSRR '06), pp. 276-281, Gaithersburg, Md, USA, August 2006.

[2] G. Arantes Jr., Rendezvous with a non-cooperating target [Ph.D. thesis], Center of Applied Space Technology, University of Bremen, Bremen, Germany, 2011.

[3] G. Arantes Jr., E. M. Rocco, I. M. da Fonseca, and S. Theil, "Far and proximity maneuvers of a constellation of service satellites and autonomous pose estimation of customer satellite using machine vision," Acta Astronautica, vol. 66, no. 9-10, pp. 14931505, 2010.

[4] D. C. Woffinden and D. K. Geller, "Navigating the road to autonomous orbital rendezvous," Journal of Spacecraft and Rockets, vol. 44, no. 4, pp. 898-909, 2007.

[5] R. M. Pinson, R. T. Howard, and A. F. Heaton, “Orbital Express advanced video guidance sensor: ground testing, flight results and comparisons," in Proceedings of the AIAA Guidance, Navigation and Control Conference and Exhibit, vol. 1, pp. 1-9, Honolulu, Hawaii, USA, August 2008.

[6] T. Weismuller and M. Leinz, "GN\&C technology demonstrated by the orbital express autonomous rendezvous and capture sensor system," in Proceedings of the 29th Annual AAS Guidance and Control Conference, vol. 1, pp. 1-9, Breckenridge, Colo, USA, 2006.

[7] R. B. Friend, "Orbital express program summary and mission overview," in Sensors and Systems for Space Applications. II, vol. 6958 of Proceedings of SPIE, 695803, Orlando, Fla, USA, April 2008.

[8] G. Arantes Jr., A. Komanduri, and L. S. Martins Filho, "Guidance for rendezvous maneuvers involving non-cooperative spacecraft using a fly-by method," in Proceedings of 21st International Congress of Mechanical Engineering (COBEM '11), Natal, Brazil, October 2011.

[9] S. A. Komanduri, Guidance and Control of a Spacecraft to Rendezvous and Dock with a Non-Cooperative Target, Center of Applied Space Technology and Microgravity ZARM, University of Bremen, Bremen, Germany, 2011. 


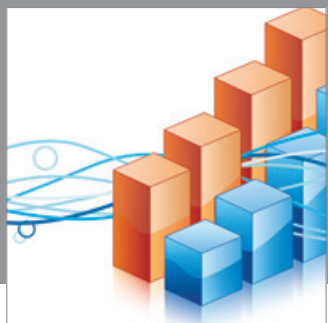

Advances in

Operations Research

mansans

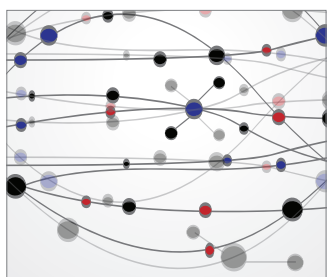

The Scientific World Journal
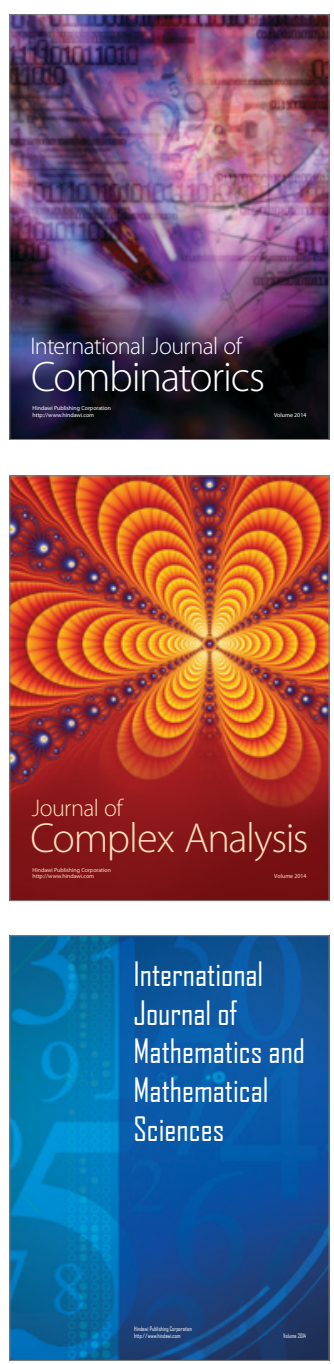
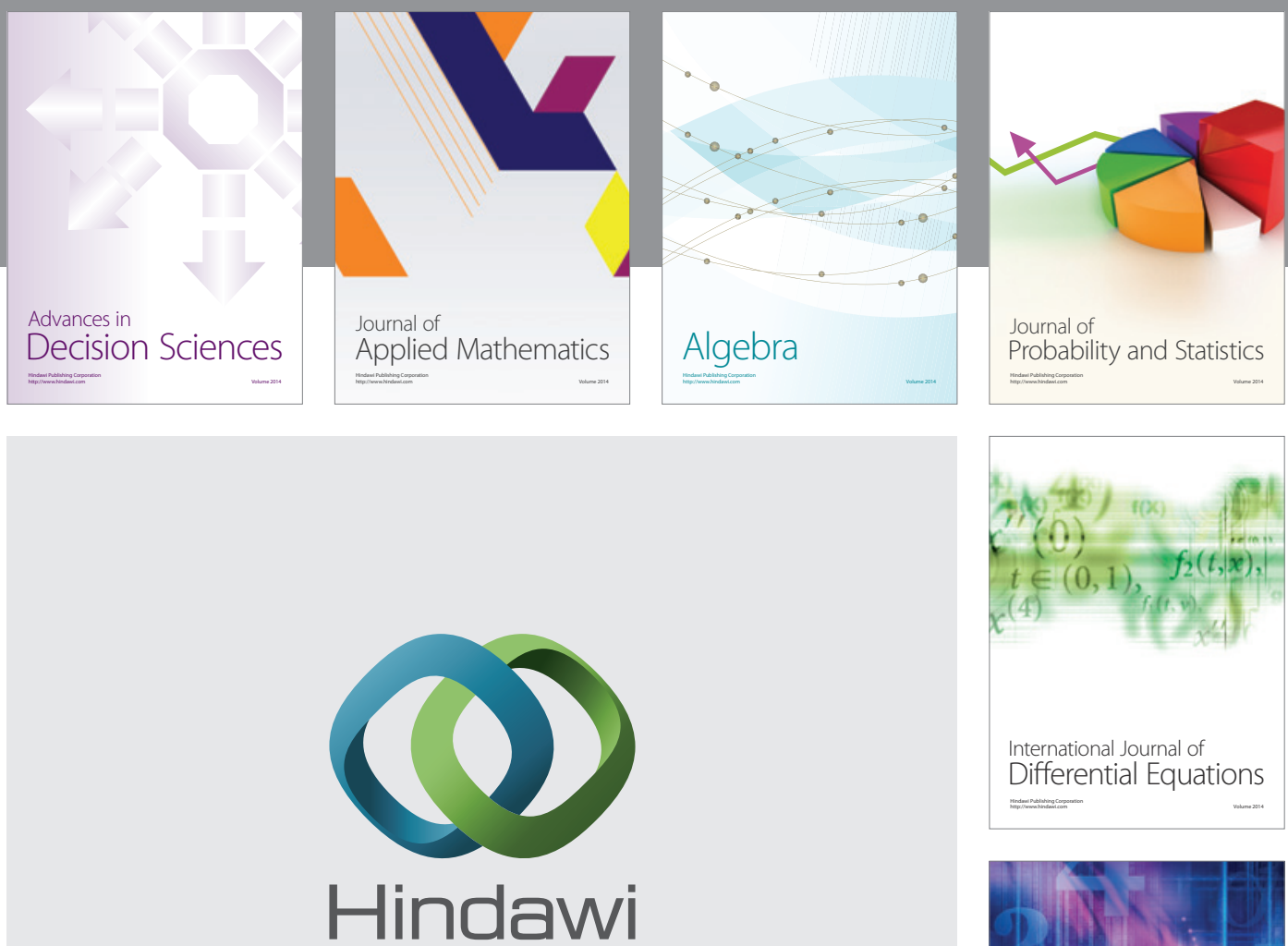

Submit your manuscripts at http://www.hindawi.com
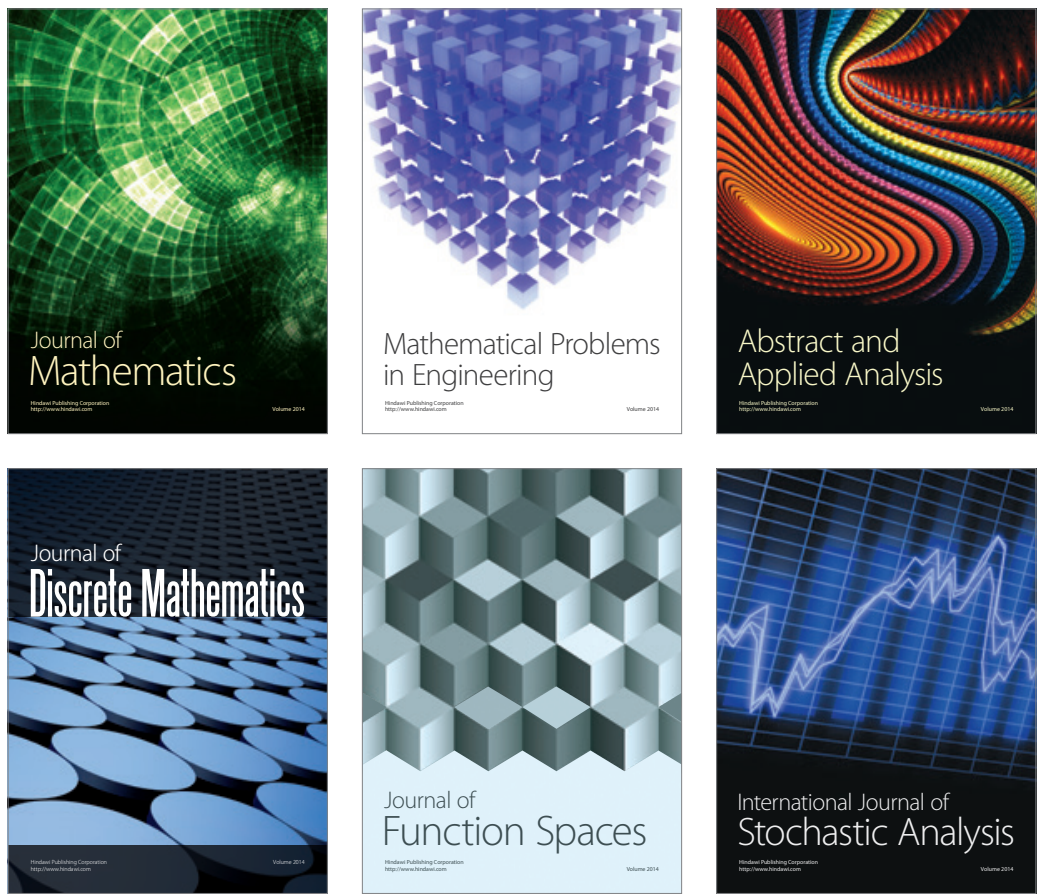

Journal of

Function Spaces

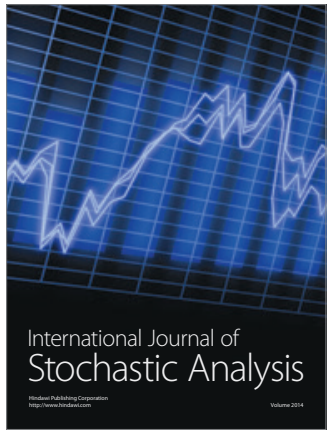

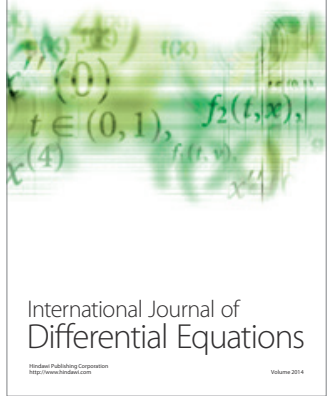
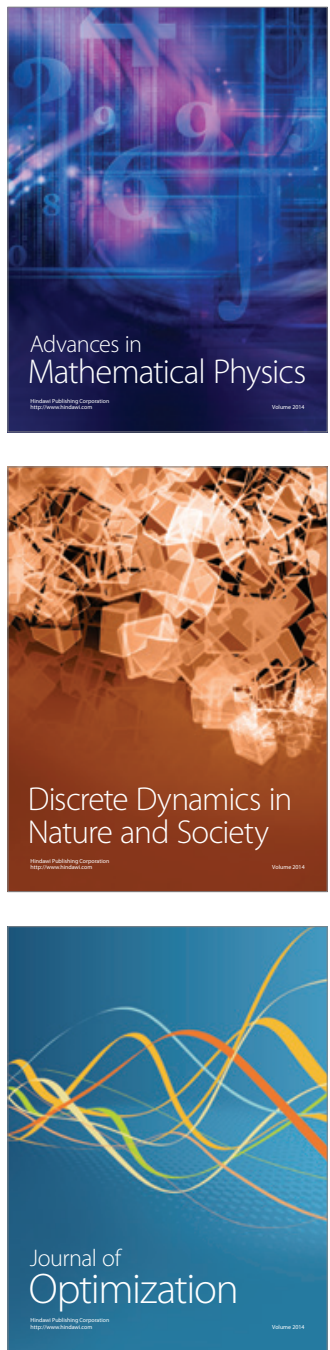